Orthopäde $2012 \cdot 41: 593-594$

DOI 10.1007/s00132-012-1905-z

Online publiziert: 25 . Juli 2012

(c) Springer-Verlag 2012

B. Wiedenhöfer $\cdot$ M. Akbar $\cdot$ B. Lehner

Department für Orthopädie, Unfallchirurgie und Paraplegiologie, Klinik für Orthopädie und Unfallchirurgie, Siftung Orthopädische Universitätsklink Heidelberg

\title{
Tumormanagement der Wirbelsäule - kein Platz für Solisten
}

Sehr geehrte Leserinnen und Leser,

die Therapie von Tumoren bedarf einer intensiven interdisziplinären Zusammenarbeit. Wesentlicher Faktor ist neben klinischen und laborchemischen Parametern eine suffiziente bildgebende Diagnostik. Die spezielle Kenntnis der Bildgebung der Wahl im Rahmen der Primärdiagnostik und der spezifischen Bildmuster ist eine ebenso unabdingbare Voraussetzung zur Bewertung der individuellen Prognose des Patienten wie die Bewertung klinischer Symptome.

Auch wenn der Anteil primärer Knochentumoren am Gesamtaufkommen von Neoplasien gering ist, ist die Auswirkung auf die Lebensqualität beträchtlich. Auch gutartige Tumoren wie Osteoidosteome beeinträchtigen durch Schmerzen und sekundäre symptomatische Deformitäten der Wirbelsäule die Lebensqualität in relevantem Ausmaß. Die Therapie dieser Tumoren war bis vor kurzer Zeit offen chirurgisch dominiert und bedeutete infolge der Resektion teilweise die Notwendigkeit der sekundären Stabilisierung durch einen Fixateur interne. Wegen der Nähe zu den neuralen Strukturen bestand eine deutliche Zurückhaltung in der Anwendung der aus der Osteoidosteomtherapie der Extremitäten bekannten Radiofrequenzablation an der Wirbelsäule. In der Zwischenzeit konnte diese stabilitätserhaltende Therapie für einen relevanten Anteil dieser Tumoren an der Wirbelsäule adaptiert werden.

Auch die Therapie primär maligner Tumoren an der Wirbelsäule konnte in einem interdisziplinären Behandlungskonzept zwischen internistisch/pädiatrischer Onkologie, Radioonkologie und Wirbelsäulenchirurgie unter Berücksichtigung von biomechanischen und Radikalitätsgesichtspunkten bedeutend weiterentwickelt werden.

Den wesentlichen Anteil der Wirbelsäulentumoren machen aber sekundäre Absiedlungen von Malignomen aus. Bis zu 70\% aller Patienten mit Malignomen entwickeln Metastasen der Wirbelsäule. Die Wirbelsäule ist damit die am häufigsten betroffene Skelettregion. Metastasenassoziierte spezifische und unspezifische Rückenschmerzen bedeuten nicht selten die symptomatische Primärmanifestation einer Tumorerkrankung. Eine adäquate Indikation zu einer operativen Therapie kann nur bei Kenntnis der neben einer operativen Therapie zur Verfügung stehenden Optionen und der zu erwartenden individuellen Prognose gestellt werden.

\section{》) Die Wirbelsäule ist die am häufigsten von Metastasen betroffene Skelettregion}

Im Laufe des letzten Jahrzehnts hat die Lebenserwartung von Patienten mit primären und sekundären Tumorabsiedlungen an der Wirbelsäule signifikant zugenommen. Diese Zunahme ist sowohl den interdisziplinären Therapiekonzepten als auch der Verbesserung und Weiterentwicklung chirurgischer Therapien und Implantate zu verdanken. Exemplarisch 
sind hier die Einführung der Zementaugmentierung zur Stabilisierung von Wirbelkörpermetastasen und die Verbreitung der Embolisation zur Blutungskontrolle stark vaskularisierter Tumoren zu nennen. Die suffiziente Stabilisierung konnte als effizientestes Mittel zur Schmerztherapie, Erhaltung der Mobilität und der Selbstständigkeit sowie Vermeidung eines Querschnittssyndroms nachgewiesen werden. Unter kurativen Ansätzen sind in ausgewiesenen Tumorzentren Verfahren wie die En-bloc-Resektion von Wirbelkörpern zur radikalen Entfernung des Tumors mit und ohne adjuvante Nachbehandlung etabliert. Mit der steigenden Lebenserwartung der Patienten sind deshalb stabilisierende chirurgische Maßnahmen von wachsender Bedeutung.

Das vorliegende Heft soll einen Überblick über die aktuellen diagnostischen und therapeutischen Optionen bei primären Tumoren und Metastasen der Wirbelsäule geben.

Unübersehbar ist in allen Artikeln, vergleichbar mit einem Orchester, das Herausstellen der Notwendigkeit einer konsequenten interdisziplinären Zusammenarbeit, ohne die auch „verbesserte chirurgische Therapieansätze "infolge schlechter Indikation „Fehltöne“ produzieren. Die Wirbelsäulenchirurgie ist in diesem Orchester zwar ungeeignet zum Solo, denn Solisten sind in diesem Orchester nicht vorgesehen. Sie sorgt aber bei geeigneter Indikation für effektive Paukenschläge mit sehr nachhaltiger Auswirkung!

Ihre

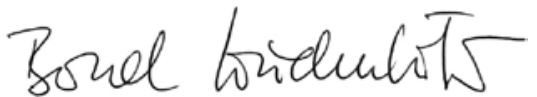

Dr. Bernd Wiedenhöfer

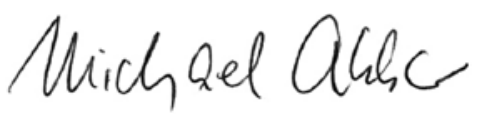

Dr. Michael Akbar

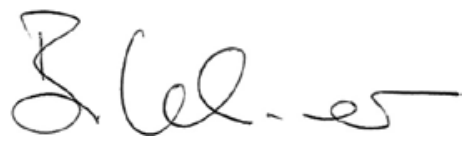

Dr. Burkhard Lehner

\section{Korrespondenzadresse}

\section{Dr. B. Wiedenhöfer}

Department für Orthopädie, Unfallchirurgie und Paraplegiologie, Klinik für Orthopädie und Unfallchirurgie, Siftung Orthopädische Universitätsklink Heidelberg

Schlierbacher Landstraße 200a,

69118 Heidelberg

bernd.wiedenhoefer@med.uni-heidelberg.de

Interessenkonflikt. Der korrespondierende Autor gibt für sich und seine Koautoren an, dass kein Interessenkonflikt besteht.

\section{AOTrauma-Seminar Göttingen \\ Frakturversorgung \\ beim Schwerverletzten \\ Wo? Wann? Wie?}

Freitag, 21. September 2012

Das diesjährige 9. Göttinger AO-Seminar legt seinen Schwerpunkt auf die Besonderheiten der Frakturversorgung beim schwerverletzten Patienten. Hier soll es ganz konkret praxisnah darum gehen, für jede einzelne Fraktur und Weichteilverletzung ein stufenweises Behandlungskonzept im Rahmen der Gesamtverletzung so früh wie möglich festzulegen. Ein großes Thema ist dabei die Frage der Primärversorgung. Früher wurden sicherlich zu viele Frakturen und Gelenkverletzungen primär versorgt, heute vielleicht zu wenige? Ein wichtiger Punkt ist auch ein klares Konzept für die Weichteildeckung.

Wissenschaftliche Leitung:

Prof. Dr. med. Klaus Michael Stürmer Direktor der Klinik für Unfallchirurgie, Plastische und Wiederherstellungschirurgie mit BG-Abteilung des BUKH Universitätsmedizin Göttingen Georg-August-Universität Robert-Koch-Straße 40, 37075 Göttingen E-Mail: ms.unfallchirurgie@med.uni-goettingen.de

Veranstaltungsort:

Sartorius College Göttingen

Otto-Brenner-Straße 20, 37079 Göttingen Phone +49 (0)551/3082101

Weitere Informationen:

Katja Rudolph

Phone +49 (0)551/39-6114

E-Mail unfallchirurgie@med.uni-goettingen.de

Anmeldung: http://goettingen1209_sem.aotrauma.org 